\title{
RESEARCH
}

Open Access

\section{Longitudinal TSPO expression in tau transgenic P301S mice predicts increased tau accumulation and deteriorated spatial learning}

Florian Eckenweber ${ }^{1}$, Jose Medina-Luque ${ }^{2}$, Tanja Blume ${ }^{1,2,3}$, Christian Sacher ${ }^{1}$, Gloria Biechele ${ }^{1}$, Karin Wind ${ }^{1}$, Maximilian Deussing ${ }^{1}$, Nils Briel ${ }^{2}$, Simon Lindner ${ }^{1}$, Guido Boening ${ }^{1}$, Barbara von Ungern-Sternberg ${ }^{1}$, Marcus Unterrainer ${ }^{1}$, Nathalie L. Albert ${ }^{1}$, Andreas Zwergal ${ }^{4,5}$, Johannes Levin ${ }^{3,5,6}$, Peter Bartenstein ${ }^{1,6}$, Paul Cumming ${ }^{7,8}$, Axel Rominger ${ }^{1,6,7}$, Günter U. Höglinger ${ }^{3,9,10}$, Jochen Herms ${ }^{2,3,6}$ and Matthias Brendel ${ }^{1,6^{*}}$ (D)

\begin{abstract}
Background: P301S tau transgenic mice show age-dependent accumulation of neurofibrillary tangles in the brainstem, hippocampus, and neocortex, leading to neuronal loss and cognitive deterioration. However, there is hitherto only sparse documentation of the role of neuroinflammation in tau mouse models. Thus, we analyzed longitudinal microglial activation by small animal $18 \mathrm{kDa}$ translocator protein positron-emission-tomography (TSPO $\mu \mathrm{PET}$ ) imaging in vivo, in conjunction with terminal assessment of tau pathology, spatial learning, and cerebral glucose metabolism.
\end{abstract}

Methods: Transgenic P301S $(n=33)$ and wild-type $(n=18)$ female mice were imaged by ${ }^{18} \mathrm{~F}-\mathrm{GE}-180$ TSPO $\mu$ PET at the ages of 1.9, 3.9, and 6.4 months. We conducted behavioral testing in the Morris water maze, ${ }^{18} \mathrm{~F}$ -

fluordesoxyglucose $\left({ }^{18} \mathrm{~F}-\mathrm{FDG}\right) \mu \mathrm{PET}$, and AT8 tau immunohistochemistry at 6.3-6.7 months. Terminal microglial immunohistochemistry served for validation of TSPO $\mu$ PET results in vivo, applying target regions in the brainstem, cortex, cerebellum, and hippocampus. We compared the results with our historical data in amyloid- $\beta$ mouse models.

Results: TSPO expression in all target regions of P301S mice increased exponentially from 1.9 to 6.4 months, leading to significant differences in the contrasts with wild-type mice at 6.4 months ( $+11-23 \%$, all $p<0.001$ ), but the apparent microgliosis proceeded more slowly than in our experience in amyloid- $\beta$ mouse models. Spatial learning and glucose metabolism of AT8-positive P301S mice were significantly impaired at 6.3-6.5 months compared to the wild-type group. Longitudinal increases in TSPO expression predicted greater tau accumulation and lesser spatial learning performance at 6.3-6.7 months.

(Continued on next page)

\footnotetext{
* Correspondence: matthias.brendel@med.uni-muenchen.de

'Department of Nuclear Medicine, University Hospital of Munich, LMU

Munich, 81377 Munich, Germany

${ }^{6}$ Munich Cluster for Systems Neurology (SyNergy), 81377 Munich, Germany

Full list of author information is available at the end of the article
}

C C The Author(s). 2020 Open Access This article is licensed under a Creative Commons Attribution 4.0 International License, which permits use, sharing, adaptation, distribution and reproduction in any medium or format, as long as you give appropriate credit to the original author(s) and the source, provide a link to the Creative Commons licence, and indicate if changes were made. The images or other third party material in this article are included in the article's Creative Commons licence, unless indicated otherwise in a credit line to the material. If material is not included in the article's Creative Commons licence and your intended use is not permitted by statutory regulation or exceeds the permitted use, you will need to obtain permission directly from the copyright holder. To view a copy of this licence, visit http://creativecommons.org/licenses/by/4.0/ The Creative Commons Public Domain Dedication waiver (http://creativecommons.org/publicdomain/zero/1.0/) applies to the data made available in this article, unless otherwise stated in a credit line to the data. 


\begin{abstract}
(Continued from previous page)
Conclusions: Monitoring of TSPO expression as a surrogate of microglial activation in P301S tau transgenic mice by $\mu$ PET indicates a delayed time course when compared to amyloid- $\beta$ mouse models. Detrimental associations of microglial activation with outcome parameters are opposite to earlier data in amyloid- $\beta$ mouse models. The contribution of microglial response to pathology accompanying amyloid- $\beta$ and tau over-expression merits further investigation.
\end{abstract}

Keywords: TSPO, PET, Tau, Microglia, P301S, Spatial learning, Glucose metabolism

\section{Introduction}

Along with features such as extracellular accumulation of amyloid- $\beta$ plaques and neuroinflammation, the intracellular aggregation of misfolded tau protein as neurofibrillary tangles (NFT) constitutes one of the neuropathological hallmarks of Alzheimer disease (AD) [1]. Under physiological circumstances, the microtubule-associated protein tau (MAPT) plays an important role in binding and stabilizing microtubules, regulating axonal transport, interacting with filaments of the cellular cytoskeleton, and probably also contributes to DNA/RNA protection in the nucleus [2]. In $\mathrm{AD}$ and non- $\mathrm{AD}$ tauopathies, though, the natively soluble and unfolded tau protein undergoes a conformational change via mechanisms such as hyperphosphorylation and misfolding, leading to diminished physiological functions of tau and its accumulation as NFT [3-5]. Deposition of hyperphosphorylated tau in brain is associated with neuroinflammation [5], which may exacerbate the ongoing tauopathy and amyloid- $\beta$ accumulation, while aggravating neuronal degeneration $[4,5]$. Indeed, the neuroinflammation in $\mathrm{AD}$ shows spatial overlap with deposition of amyloid- $\beta$ and NFT accumulation [6]. Furthermore, particular components of the neuroinflammatory cascade promote the development of NFT [7]. Importantly, the onset of neuroinflammation occurs early in tauopathies, suggesting that biomarkers of neuroinflammation might serve as a tool to predict the individual disease course [8].

The transgenic P301S mouse model accumulates tau in the brainstem [9-11], hippocampus [10, 12], and cerebral cortex [10], and this accumulation is accompanied by a decline in spatial learning [12]. Immunohistochemical (IHC) analysis revealed increased microglial activation in transgenic P301S mice at 5 months of age, compared to findings in wild-type mice [13]. Other studies demonstrate the capacity of wild-type mouse microglia to phagocytize NFTs accumulating in the brain of P301S mice [14] and likewise in cultured neurons from P301S mice [15], consistent with a dual role of microglial activation in exerting neuroprotective [14] and neurodegenerative effects [15]. However, the time course of microglial neuroinflammation and its net effect on neurodegeneration is not yet established in this mouse model of tauopathy.
Because understanding the role of neuroinflammation in $\mathrm{AD}$ and non-AD tauopathies is of crucial importance, we undertook longitudinal monitoring of microglial activation in P301S mice by means of ${ }^{18} \mathrm{~F}$-GE-180 $\mu$ PET in vivo, extending a technique we have established in amyloid- $\beta$ mouse models. The tracer ${ }^{18} \mathrm{~F}$-GE-180 binds to the $18 \mathrm{kDa}$ translocator protein (TSPO) expressed in activated microglial cells in living mouse brain, showing excellent correlation with ex vivo validation in several different amyloid- $\beta$ mouse models [16-19]. We now aimed to test the predictive value of early microglial activation in this tau mouse model by undertaking serial ${ }^{18} \mathrm{~F}$-GE-180 $\mu$ PET until 6.4 months of age, augmented by analyses of spatial learning with the Morris water maze test (MWM) and glucose metabolism with ${ }^{18} \mathrm{~F}$-fluorodesoxyglucose $\left({ }^{18} \mathrm{~F}\right.$-FDG) $\mu$ PET. Finally, we made an IHC examination of tau and microglia by AT8, IBA1, and CD68 antisera. Moreover, we compared the temporal kinetics of microglial activation of the tau mouse model with corresponding findings retrieved from our historical amyloid- $\beta$ mouse model studies.

\section{Materials and methods}

\section{Animals and study design}

All experiments were performed in compliance with the National Guidelines for Animal Protection, Germany, and with the approval of the regional animal committee (Regierung Oberbayern) and were overseen by a veterinarian. Animals were housed in a temperature- and humidity-controlled environment with $12 \mathrm{~h}$ light-dark cycle, with free access to food (Sniff, Soest, Germany) and water. $\mu$ PET experiments were carried out in homozygous female human tau P301S mice $(n=33)$, a mouse line expressing the human $0 \mathrm{~N} 4 \mathrm{R}$ tau isoform with the P301S mutation in exon 10 of the MAPT gene under control of the murine thy1 promoter [10], whereas control studies were conducted in age and sex matched wild-type (WT, $n=18$ ) mice. TSPO $\mu$ PET examinations were performed in a longitudinal design at baseline (1.9 months of age) and two follow-up measurements (3.9 and 6.4 months of age) (Fig. 1a). ${ }^{18}$ F-FDG $\mu$ PET scans were conducted at the age of 6.4-6.5 months. The MWM test was administered at $12 \pm 7$ days before the 


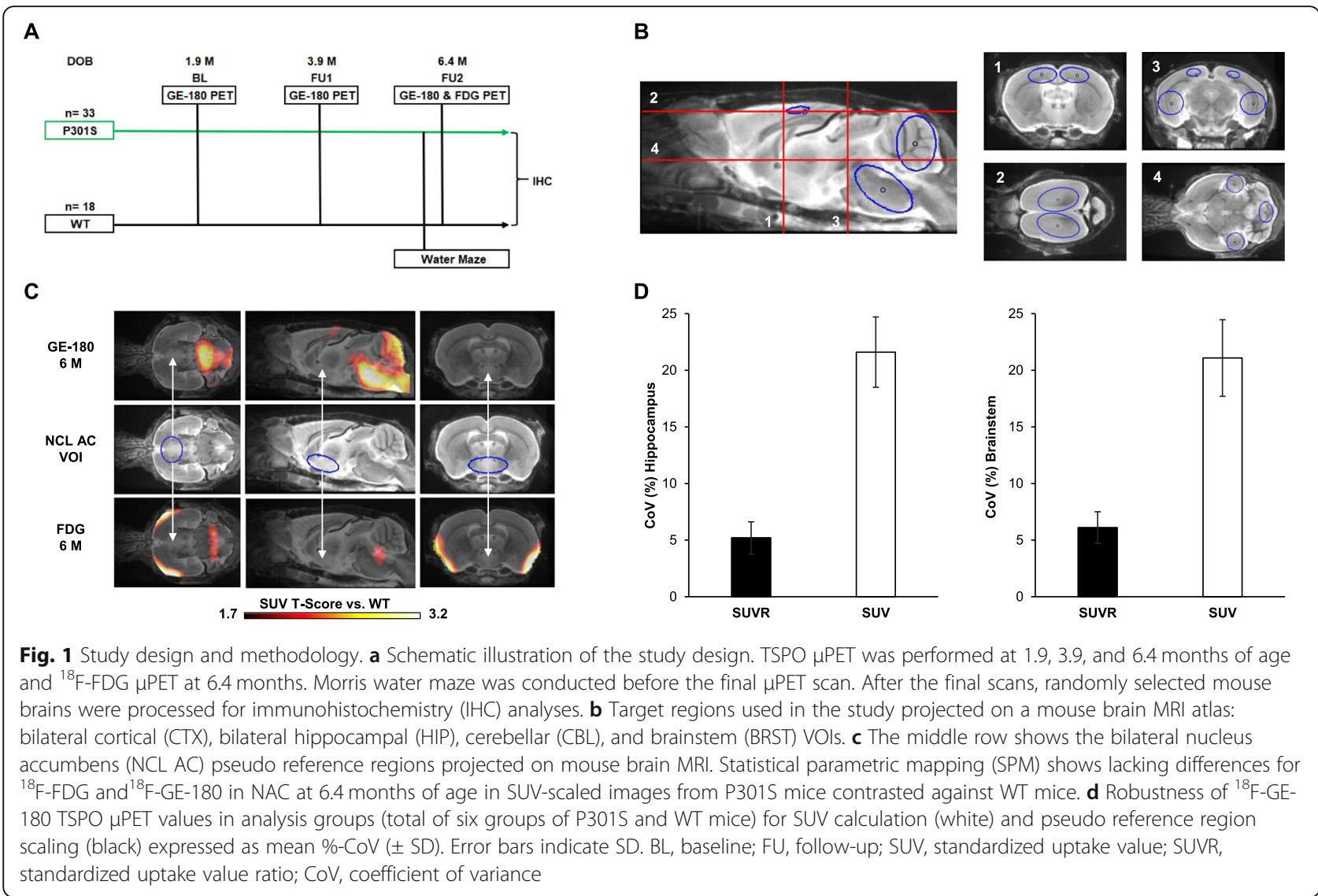

final TSPO $\mu$ PET scan in P301S $(n=22)$ and WT $(n=18)$ mice. After recovery of 2-6 days following the final $\mu \mathrm{PET}$ scans, randomly selected brains from P301S $(n=14)$ mice and WT $(n=5)$ mice were processed for IBA1, CD68, and AT8 IHC in the brainstem and cortex. Additional IHC analyses were conducted in small subgroups $(n=3)$ of P301S mice and $(n=2)$ WT mice at 2.7 and $4.8 / 4.5$ months of age. Mice intended for IHC were deeply anaesthetized prior to transcardial perfusion with saline followed by $4 \%$ paraformaldehyde and subsequent brain extraction. Brains were then fixed by immersion in $4 \%$ paraformaldehyde at $4{ }^{\circ} \mathrm{C}$ for $10 \mathrm{~h}$ and then transfered to phosphate buffered saline (PBS). Samples were stored in PBS with $0.01 \%$ sodium azide at $4{ }^{\circ} \mathrm{C}$ until preparation for staining. Representative $50 \mu \mathrm{m}$ thick slices per animal were then cut in the axial plane using a vibratome (VT 1000 S, Leica, Wetzlar, Germany). We reprocessed historical $\mu$ PET ${ }^{18} \mathrm{~F}-\mathrm{GE}-180$ scans from amyloid- $\beta$ APP/PS1 [19] and $A p p^{N L-G-F}$ mice [20] for comparison of their longitudinal microglial activation with present findings associated with tau accumulation in P301S mice.

\section{Radiochemistry and $\mu$ PET Imaging}

Radiosynthesis of ${ }^{18} \mathrm{~F}$-GE-180 was performed as previously described [16], and ${ }^{18}$ F-FDG was purchased commercially. $\mu$ PET imaging was described as reported previously [16]. In brief, all mice were anesthetized with isoflurane $(1.5 \%$, delivered at $3.5 \mathrm{~L} / \mathrm{min})$ and were placed in the aperture of the Siemens Inveon DPET. ${ }^{18} \mathrm{~F}-\mathrm{GE}$ $180 \mathrm{TSPO} \mu \mathrm{PET}$ with an emission window of $60-90 \mathrm{~min}$ p.i. was used to measure cerebral TSPO expression, and (on another day) ${ }^{18}$ F-FDG $\mu$ PET with an emission window of 30-60 min p.i. was used for assessment of cerebral glucose metabolism.

\section{$\mu$ PET image analysis}

All analyses were performed using PMOD (V3.5, PMOD technologies, Basel, Switzerland). After co-registration to an MRI mouse atlas [17], we conducted intensity normalization of images to standardized uptake value (SUV) relative to uptake in the myocardium for ${ }^{18} \mathrm{~F}$-GE$180 \mu \mathrm{PET}[21]$ and by conventional SUV calculation for ${ }^{18}$ F-FDG $\mu$ PET. Predefined bilateral cortical (CTX, 24 $\mathrm{mm}^{3}$ ), bilateral hippocampal (HIP, $11 \mathrm{~mm}^{3}$ ), cerebellum $\left(\mathrm{CBL}, 12 \mathrm{~mm}^{3}\right)$, and brainstem (BRST, $12 \mathrm{~mm}^{3}$ ) target volumes of interest (VOIs) were applied for both tracers (Fig. 1b). We selected these target VOIs based on our immunohistochemical findings of tau accumulation in our earlier studies of the P301S mouse model [9-12]. 
Voxel-based comparisons of SUV maps between groups of P301S and WT mice were performed using statistical parametric mapping (SPM, described below) to identify first a suitable reference tissue for $\mu$ PET quantification. Here, this criterion is met by any brain region in which tracer uptake did not differ with genotype or age, either for ${ }^{18} \mathrm{~F}-\mathrm{FDG}$ or for ${ }^{18} \mathrm{~F}-\mathrm{GE}-180$. Our analysis showed that the bilateral nucleus accumbens $\left(\mathrm{NAC}, 10 \mathrm{~mm}^{3}\right.$ ) served adequately as a pseudo reference region for calculation of SUV-ratio (SUVR) values for both $\mu$ PET tracers (Fig. 1c). We next calculated target-to-reference tissue SUVRs, i.e., $\mathrm{SUVR}_{\mathrm{CTX} / \mathrm{NAC}}$, SUVR $_{\text {HIP/NAC, }}$ SUVR CBL/NAC, and SUVR $_{\text {BRST/NAC, for }}$ ${ }^{18} \mathrm{~F}$-GE-180 and ${ }^{18} \mathrm{~F}$-FDG $\mu$ PET. To quantify longitudinal changes in microglia activation, the percentage change between SUVR at baseline and the last followup scan was calculated. To allow longitudinal analysis of combined regions of interest, the area under the curve (AUC) of ${ }^{18} \mathrm{~F}-\mathrm{GE}-180 \mu \mathrm{PET}$ SUVR between baseline and the terminal imaging time point was calculated as previously described [22].

\section{SPM analysis}

For both tracers, whole-brain voxel-wise comparisons of SUV and SUVR images between groups of P301S and WT mice were performed by SPM using SPM8 routines (Wellcome Department of Cognitive Neurology, London, UK) as previously established in our group [9]. This analysis was implemented in MATLAB (version 7.1), as adapted from Sawiak et al. [23] for mouse data. We performed two-sample $t$ tests, setting a significance threshold of $p<0.05$, uncorrected for multiple comparisons.

\section{Behavioral testing}

$n=22$ P301S and $n=18$ WT mice were subjected to a MWM test for spatial learning and memory deficits, which was performed according to a standard protocol [20]. On training days one through five, each mouse had to perform four trials per day in the test basin, with maximum time set to $70 \mathrm{~s}$. The test trial was performed on day six. For analyses of escape latency and distance during MWM testing, we used the video tracking software EthoVision ${ }^{\circ}$ XT 13 (Noldus).

\section{Immunohistochemistry}

A standard free-floating immunofluorescence (IF) protocol was performed in the cortex and brainstem areas matching the $\mu$ PET brain regions. As previously described [9, 17], fixed $20-\mu \mathrm{m}$ thick brain sections were first rinsed either overnight or for $48 \mathrm{~h}$ in PBS with $0.2 \%$ Triton X-100 containing one following primary antibodies: rabbit monoclonal IBA1 (1:500. Wako:19-19741), mouse monoclonal phospho-Tau (Ser202, Thr2015
(AT8), 1:500. Thermofisher: MN1020), and rat monoclonal CD68 (1:500. Bio-rad: MCA1857). After washing in $\mathrm{PBS}$, sections were then incubated in a combination of three secondary antibodies (Alexa 488 goat anti-rabbit, Alexa 594 goat anti-mouse and Alexa 647 goat anti-rat IgG). For long-term preservation, the labelled slices were mounted in DAKO fluorescence mounting medium. Analyses for overall density (OD) and area coverage (area-\%) were carried out by using the free access program ImageJ (https://imagej.nih.gov/ij/). The measurements were performed by using images obtained from a confocal microscope (LSM 780 Axio invers). Acquisitions were performed at $\times 20$ and $\times 40$ objectives in at least three sagittal sections. The implemented threshold tool was used for to limit the extracted data to the specific signal of the primary antibodies (IBA1, CD68, or AT8). To subtract unspecific signal from the OD, background staining signal, as obtained from immunonegative regions, was deducted. OD per region was calculated for IBA1 and CD68 whereas the area-\% was calculated for AT8.

\section{Statistics and calculations}

Statistical analyses were performed in SPSS (Version 25, IBM Deutschland GmbH, Ehningen, Germany). The Kolmogorov-Smirnov test served to evaluate normal distribution of all data. Coefficients of variation $(\mathrm{CoVs})$ were calculated as a measure of robustness in group data.

Two-tailed unpaired $t$ tests were used to compare intergroup readouts $\left({ }^{18} \mathrm{~F}\right.$-FDG $\mu$ PET, MWM, and IHC) of P301S and age-matched WT mice for all normally distributed readouts. For intergroup comparison of nonnormally distributed readouts, Mann-Whitney $U$ tests were calculated. Significance levels for intergroup comparison of longitudinal TSPO $\mu$ PET were calculated by a mixed model repeated measures ANOVA. Effect sizes between P301S and WT were determined as Cohen's $d$. For correlation analyses, Pearson's coefficients of correlation $(R)$ were calculated for normally distributed readouts. For non-normally distributed readouts, Spearman's coefficients of correlation $\left(r_{\mathrm{S}}\right)$ were calculated. Cortical TSPO $\mu$ PET values in P301S were transformed into $z$ scores relative to WT findings as described previously and then plotted as a function of age. Our historical cortical TSPO $\mu$ PET data of APP/PS1 $(n=17)$ [19] and $A p p^{N L-G-F}(n=21)$ [20] mice were reprocessed in the same way. Staining intensity as a function of age was likewise calculated for the current AT8 data in P301S mice and earlier findings of ${ }^{18} \mathrm{~F}$-florbetaben $z$-score data in two historical amyloid- $\beta$ mouse models. For TSPO $\mu \mathrm{PET}$, we calculated the AUC of $z$-scores for all mice with successful completion of three or more serial $\mu \mathrm{PET}$ scans. AUC values were adjusted for the maximum $z$ - 
score in the studied period, normalized to the observation time, and compared between tau and amyloid- $\beta$ mice by ANOVA with Tukey post hoc correction. A validation analysis was performed for AUC within the same age range (2-7 months) of all three models. Division by the final TSPO $z$-score was omitted in this analysis due to limited serial data points. A threshold of $p<0.05$ was considered to be significant for rejection of the null hypothesis.

\section{Results}

TSPO $\mu$ PET facilitates monitoring of microglial activation in P301S mice

We first established that microglial activation can be monitored by longitudinal TSPO $\mu$ PET imaging in P301S mice. To obtain robust TSPO $\mu$ PET measures, we validated a suitable pseudo reference tissue: among the various possible regions, SUVR scaled TSPO $\mu$ PET values in the nucleus accumbens (SUVR $\mathrm{NAC}_{\mathrm{C}}$ ) showed more robust group results when compared to conventional SUV scaling, as indicated by lower CoVs. For instance, SUVR $\mathrm{NAC}_{\mathrm{N}} \mathrm{CoVs}$ in the brainstem were $6 \pm 1 \%$ (range, 4 to $7 \%$ ) whereas the corresponding SUV CoVs were $21 \pm 3 \%$; (range, 16 to $27 \%$; Fig. $1 \mathrm{~d}$ ).

${ }^{18} \mathrm{~F}-\mathrm{GE}-180$ SUVR indicated exponential increases with age for all four target regions in P301S mice and significantly weaker comparable increases in WT mice. At 6.4 months, where was significantly elevated TSPO $\mu$ PET signal in the cortex $\left(+12 \%, p=1.0 \mathrm{E}^{-6}\right.$, Cohen's $\left.d=1.84\right)$, the hippocampus $\left(+11 \%, p=5.2 \mathrm{E}^{-11}\right.$, Cohen's $\left.d=2.85\right)$, the brainstem $\left(+23 \%, p=3.4 \mathrm{E}^{-14}\right.$, Cohen's $\left.d=3.74\right)$, and the cerebellum $\left(+18 \%, p=8.6 \mathrm{E}^{-13}\right.$, Cohen's $\left.d=3.25\right)$ of P301S mice when compared to WT (Fig. 2a). Voxel-wise analyses at 6.4 months mirrored this finding for ${ }^{18} \mathrm{~F}-\mathrm{GE}$ 180 , with the most pronounced elevation in the hindbrain of P301S mice when compared to WT. However, SPM analysis revealed the first onset of increased ${ }^{18} \mathrm{~F}-\mathrm{GE}-180$ SUVR in P301S mice at 3.9 months of age (Fig. 2b). IHC with the microglial markers IBA1 and CD68 validated in vivo findings by indicating a similar increase of microglial activation with age in P301S mice when compared to increases of TSPO PET. Differences between P301S and WT mice at 6.6-6.7 months of age in IHC were also similar to TSPO $\mu$ PET for the cortex (IBA1: $+39 \%, p=0.011$,

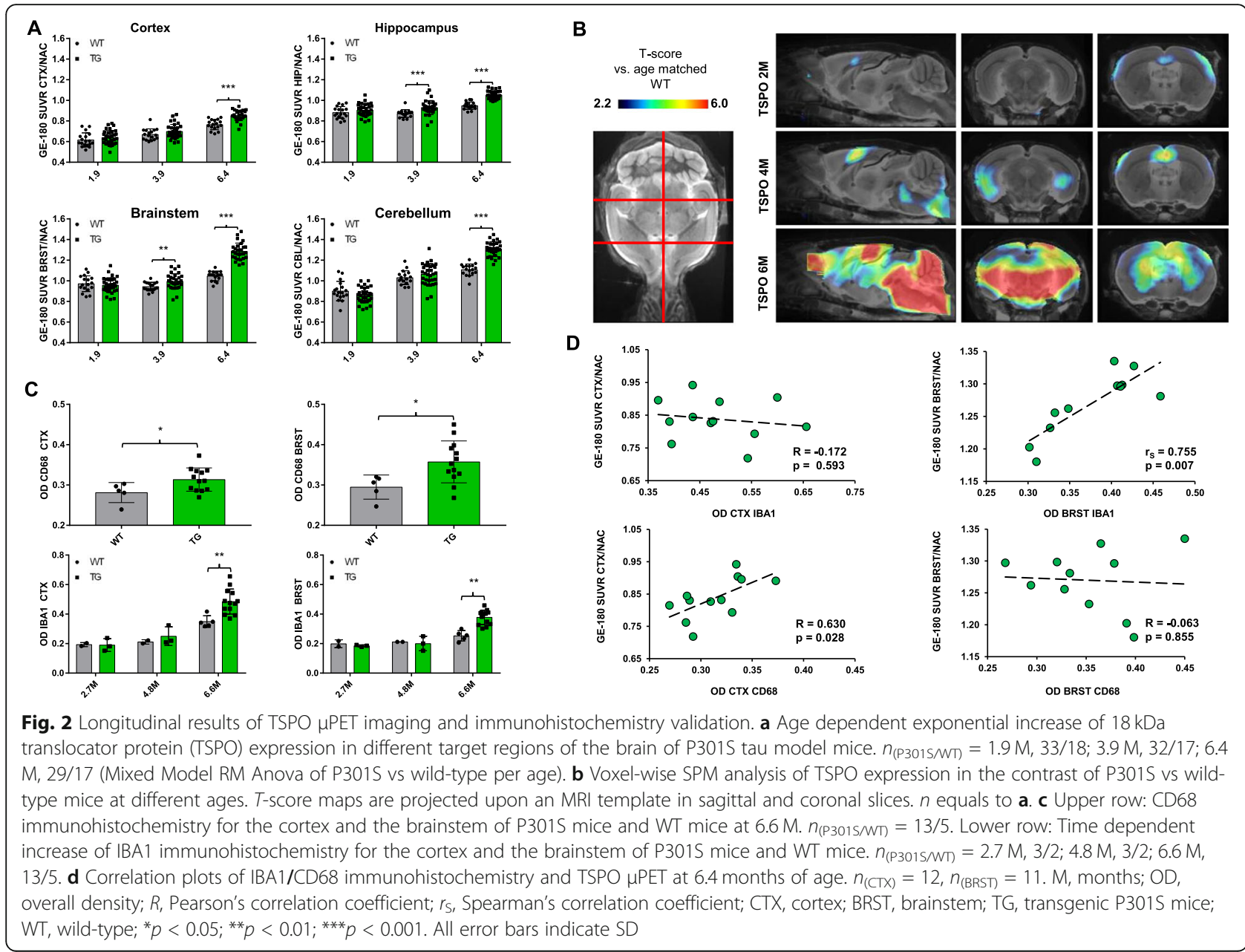


Cohen's $d=2.16 /$ CD68: $+12 \%, p=0.043$, Cohen's $d=$ 1.29; Fig. 2c) and brainstem (IBA1: $+50 \%, p=1.7 \mathrm{E}^{-4}$, Cohen's $d=3.12 /$ CD68: $+21 \%, p=0.023$, Cohen's $d=$ 1.56). The TSPO $\mu \mathrm{PET}$ signal in the cortex correlated significantly with the IHC for the phagocytosis marker CD68 $(R=0.630, p=0.028)$, but not with IBA1 $(R=-0.172, p=$ $0.593)$ as a general marker of microglial activation, whereas the TSPO $\mu$ PET signal in the brainstem correlated with IBA1 $\left(r_{\mathrm{S}}=0.755, p=0.007\right)$ but not with CD68 $(R=-0.063, p=0.854$; Fig. $2 \mathrm{~d})$. Table 1 provides a summary of TSPO $\mu$ PET and microglia IHC results. Supplemental Figure 1 shows IBA1 and CD68 staining at different ages.

\section{Longitudinal microglial response in P301S mice is attenuated and delayed when compared to amyloid- $\beta$ mouse models}

Next, we asked if there are differences in the longitudinal development of microglial activation between the present tau mice and earlier studies in amyloid- $\beta$ mouse models. To this end, we calculated standardized differences of TSPO $\mu \mathrm{PET}$ in the cortex of P301S, APP/PS1, and $A p p^{N L-G-F}$ mice in comparison to WT and compared TSPO $\mu$ PET as a function of age between models. Standardized differences were lower in P301S mice when compared to both amyloid- $\beta$ mouse models (Fig. $3 \mathrm{a}-\mathrm{c}$ ). Longitudinal cortical TSPO $\mu$ PET increases in the P301S mice followed a convex quadratic function $\left(y=0.09 x^{2}-\right.$ $0.37 x+1.18)$ whereas cortical TSPO $\mu$ PET increases in amyloid- $\beta$ mouse models were characterized by concave quadratic functions $\left(A p p^{N L-G-F}: y=-0.10 x^{2}+2.16 x-\right.$ 4.53; APP/PS1: $y=-0.04 x^{2}+1.21 x-1.23$; Fig. $3 \mathrm{a}-\mathrm{c}$ ). The TSPO $\mu$ PET AUCs integrated over time were significantly higher in amyloid- $\beta$ mouse models $\left(A p p^{N L-G-F}\right.$, $0.79 \pm 0.25 ; \mathrm{APP} / \mathrm{PS} 1,0.89 \pm 0.41)$ when compared to P301S mice $(0.52 \pm 0.25 ; p=0.021 / p=0.016)$ after adjustment for the maximum increase at the ultimate age and normalization to the observation period (Fig. 3d). Importantly, there were concave quadratic increases over time for both tau to AT8 staining in P301S $\left(y=-0.10 x^{2}\right.$ $+1.69 x-1.29)$ and fibrillar $A \beta$ to ${ }^{18} \mathrm{~F}$-florbetaben $\mu \mathrm{PET}$ imaging in APP/PS1 $\left(y=-0.04 x^{2}+1.14 x-3.22\right)$ and $A p p^{N L-G-F}\left(y=-0.04 x^{2}+0.86 x-2.67\right)$ mice. The results of the full observation periods were validated by a direct comparison of similar age ranges between all models (Fig. 3e-h).

\section{Longitudinal microglial activation is associated with poor outcome parameters in P301S mice}

Finally, we endeavored to test if baseline or longitudinal TSPO $\mu$ PET can predict neuropathological, behavioral, and functional outcome measures in P301S mice. To this end, we recorded tau quantification, a spatial learning paradigm, and ${ }^{18} \mathrm{~F}$-FDG $\mu \mathrm{PET}$ at the terminal time- point and correlated these three endpoints with baseline and longitudinal TSPO $\mu$ PET readouts. AT8 positive tau accumulation was confirmed by immunohistochemistry, which showed regional colocalization with activated microglia in the cortex and the brainstem (Fig. 4a). P301S mice aged 6.3 months took twice as long as WT mice to find the virtual platform in MWM (P301S $39 \pm$ $20 \mathrm{~s}$; WT $20 \pm 21 \mathrm{~s} ; p=3.4 \mathrm{E}^{-4}$ ) and revealed a lower frequency of crossing the platform area (P301S $1.5 \pm 1.2$ times, WT $3.3 \pm 2.3$ times; $p=0.010$ ). Swim speed of WT mice was significantly faster when compared to P301S mice $\left(21 \pm 2 \mathrm{~cm} / \mathrm{s}\right.$ vs $\left.14 \pm 3 \mathrm{~cm} / \mathrm{s}, p=4.8 \mathrm{E}^{-8}\right)$. Therefore, the travelled distance was additonally analyzed as a readout unbiased from locomotor problems. Significantly longer travelled distance in P301S mice when compared to WT $(544 \pm 268 \mathrm{~cm}$ vs $404 \pm 414 \mathrm{~cm}$, $p=0.019)$ validated the deficits in spatial memory independent from beginning locomotor problems (Fig. 4b). ${ }^{18}$ F-FDG $\mu$ PET revealed significantly decreased glucose metabolism of P301S mice compared with WT mice at 6.4-6.5 months of age in the hippocampus $(-6 \% ; p=$ $\left.1.9 \mathrm{E}^{-4}\right)$ and the brainstem $(-4 \% ; p=0.002)$, which was also mirrored by voxel-wise analyses (Fig. 4c).

Longitudinal increases of the TSPO $\mu$ PET signal were positively correlated with AT8 accumulation in the cortex $(R=0.570, p=0.033)$ and in the brainstem $(R=$ 0.681, $p=0.007$, Fig. 5a). Baseline TSPO $\mu$ PET did not significantly predict tau accumulation.

Higher TSPO $\mu$ PET values over time in the cortex and the hippocampus of P301S mice were associated with worse performance in MWM at the late stage (escape latency: $r_{\mathrm{S}}=0.493 ; p=0.038$; frequency: $r_{\mathrm{S}}=-0.258, p=$ 0.302; Fig. $5 b)$. Baseline TSPO $\mu$ PET did not significantly predict MWM readouts at 6.3 months of age.

Baseline TSPO $\mu$ PET elevation in the cortex $(R=-$ $0.448 ; p=0.028)$ and in the hippocampus $(R=-0.415$; $p=0.044)$ of P301S mice at 1.9 months of age predicted stronger hypometabolism in FDG $\mu$ PET at 6.4-6.5 months of age (Fig. 5c). On the other hand, longitudinal increases of the TSPO $\mu$ PET signal in these regions were associated with elevated glucose metabolism or predicted a trend towards elevated glucose metabolism at 6.4-6.5 months of age (Fig. 5c).

\section{Discussion}

We report the first longitudinal in vivo $\mu$ PET imaging study of microglial activation together with assessment of multiple outcome parameters in a tau mouse model. Our data clearly indicate that $\mu \mathrm{PET}$ with the TSPO tracer ${ }^{18} \mathrm{~F}$-GE-180 gives reliable assessment of microglial activation in living P301S mice, as proven by the high correlation with specific IHC markers. Analysis of individual TSPO $\mu$ PET time courses to 6.4 months of age revealed that microglial activation in the tau model mice 


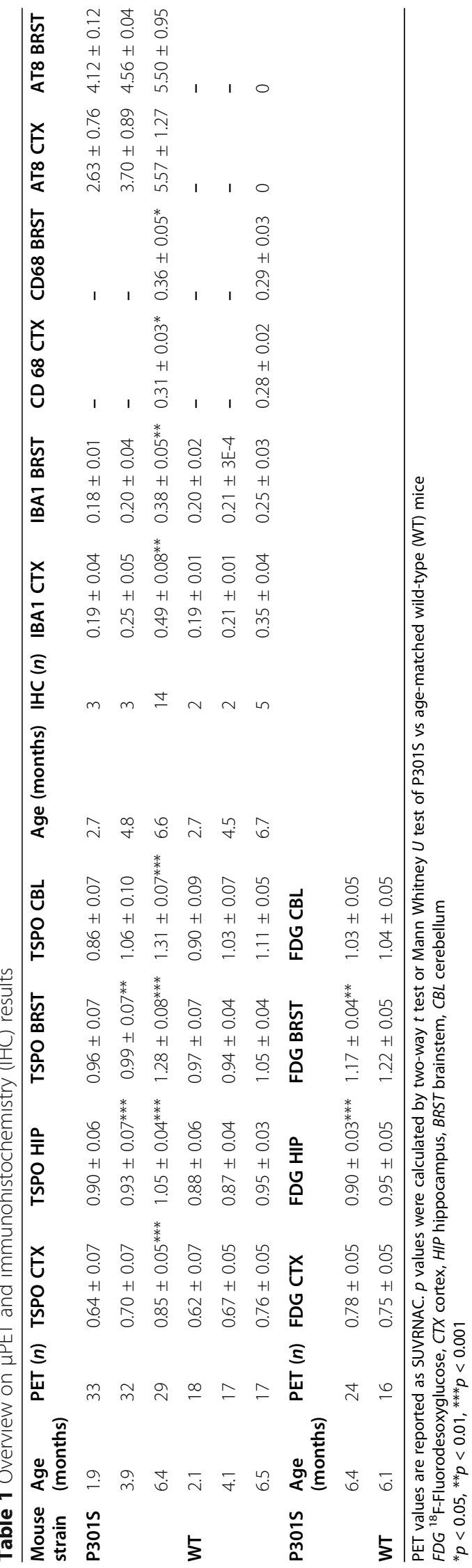




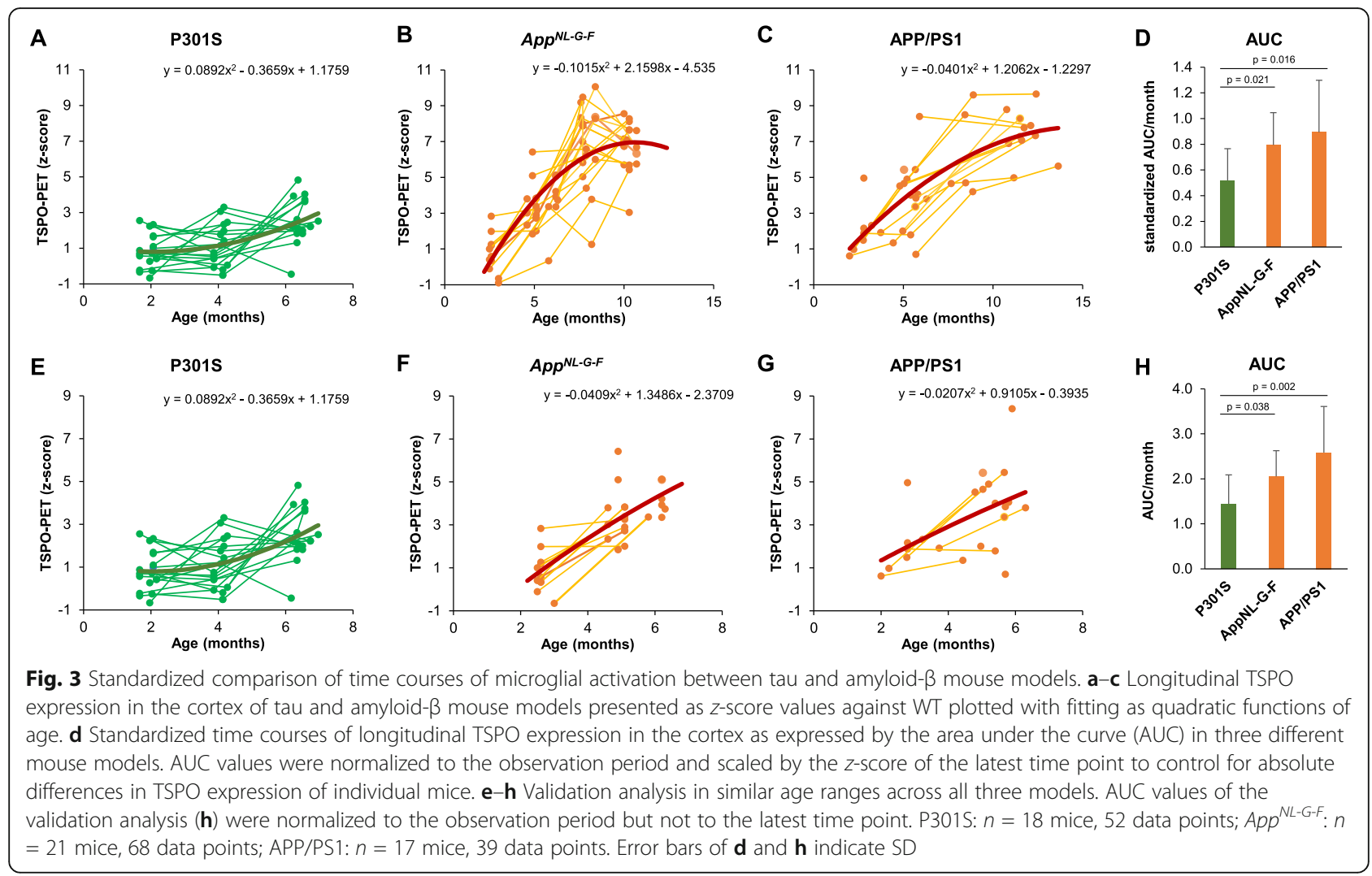

exponentially increases with age. Importantly, longitudinal elevations of TSPO expression in P301S mice predicted aggravated tau accumulation and worse performance in spatial learning. Levels of glucose metabolism at the late stage were positively associated with longitudinal TSPO $\mu$ PET increases, but early elevations of TSPO expression predicted stronger hypometabolism in P301S mice. These findings may be reconciled by consideration of the ambivalent role of microgliosis in neurodegeneration, in some circumstances imparting neuroprotection, and in other circumstances marking a more aggressive pathology. This dual role is seemingly decided by the type of pathology (i.e., tau or amyloid- $\beta$ over-expression) and the time course.

We show that transfer of TSPO $\mu$ PET technology from different amyloid- $\beta$ mouse models $[19,22,24]$ to the present tau mouse model is feasible without major caveats. As in some former studies [16, 20], we successfully validated a suitable pseudo reference region for TSPO $\mu$ PET in P301S mice, and we were again able to show that this methodology reduces variance at the group level. This SUVR approach supported the detection of robust longitudinal increases of TSPO expression in different target regions of the P301S mouse model, which were matched with increases of microglial activation markers measured later by IHC. Furthermore, our $\mu$ PET data were validated by direct correlation with IHC at the terminal time point. Interestingly, the phagocytosis marker $\mathrm{CD} 68$, which indicated the highest association with TSPO $\mu$ PET in our earlier study of amyloid- $\beta$ mice [19], significantly correlated with TSPO $\mu$ PET values in the cortex but not in the brainstem of tau mice. Yet, the present correlation of ${ }^{18} \mathrm{~F}$-GE-180 TSPO $\mu$ PET with the more general activation marker IBA1 in the brainstem of tau mice could hinge on different microglia phenotypes and their covariance with TSPO expression in different brain regions [25]. However, we note that several specific and random factors like regional blood flow or slice selection could also cause these divergent observations. Our findings are in line with those of a study conducting longitudinal $\mu \mathrm{PET}$ with the TSPO tracer ${ }^{11} \mathrm{C}-\mathrm{AC}-5216$ in another tau mouse model (PS19), likewise showing time dependent progression of TSPO expression in the entorhinal cortex and the hippocampus [26]. Another study of PS19 mice found microglial activation especially in the hippocampus to occur ahead of discernible tau accumulation and brain atrophy [27]. This implicates that microglial activation can already be present in very early phases of $\mathrm{AD}$, when seeding of tau just begins and is not identifiable by tau IHC yet. This also suggests that neuroinflammation could be triggered by non fibrillar components of tau, ending up in a vicious circle in the pathogenesis of AD. However, we note that many of the complex underlying mechanisms 

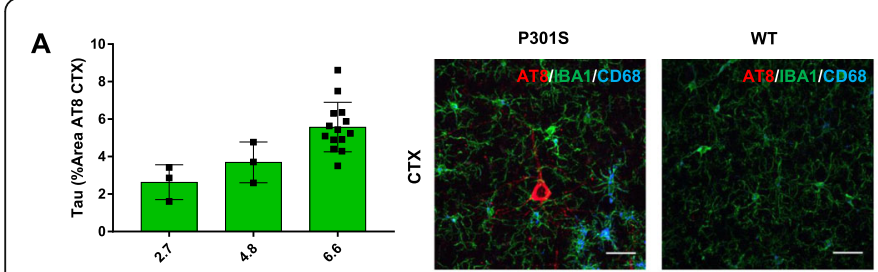

B
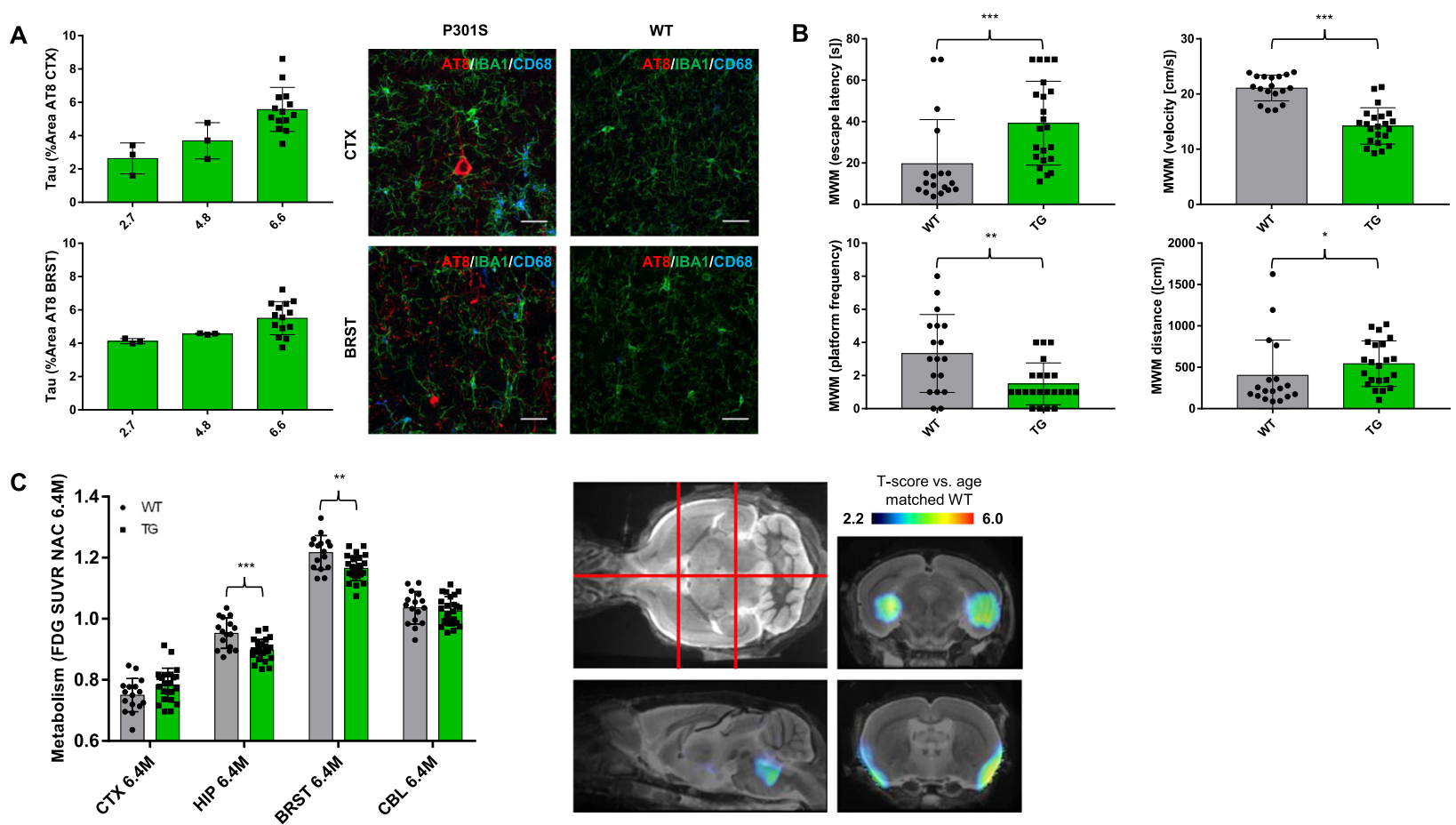

Fig. 4 Outcome parameter in comparison of P301S and wild-type mice. a Longitudinal accumulation of tau measured via AT8 immunohistochemistry and representative images of co-staining with IBA1 and CD68 in the cortex (upper row) and brainstem (lower row). n (2.7 $\mathrm{M} / 4.8 \mathrm{M})=3 ; n(6.6 \mathrm{M})=14$. Scale bar $=30 \mu \mathrm{m}$. b Performance of mice in Morris water maze (MWM) at study termination with escape latency, velocity, swimming distance, and frequency of platform crossing. $n=22$ P301S, $n=18$ WT. c Glucose metabolism (FDG-PET uptake) in the different target VOls at 6.4 months of age and SPM results of the contrast P301S and wild-type (WT) mice. $n=24$ P301S, $n=16$ WT. Color coding shows regions with decreased glucose metabolism (T-scores) in P301S mice when compared to WT upon an MRI template in sagittal and coronal slices, as indicated by red lines in the axial MRI slice. CTX, cortex; HIP, hippocampus; BRST, brainstem; CBL, cerebellum; M, months; SUVR, standardized uptake value ratio; ${ }^{*} p<0.05 ;{ }^{* *} p<0.01 ;{ }^{* *} p<0.001$. All error bars indicate SD

and interrelations are not completely discovered yet [3, $5,28]$. Understanding the role of neuroinflammation in neurodegenerative disorders is of high importance as it is associated with deposition of NFT, amyloid- $\beta$, and neuronal degeneration. Translational imaging in mouse models and patients will facilitate to close important research gaps in terms of reciprocal validation and therapy monitoring.

As there have been no direct comparisons of the time courses of microglial activation between amyloid- $\beta$ and tau mouse models, we put a special focus on contrasting longitudinal in vivo TSPO expression of P301S mice against existing data in two common amyloid- $\beta$ mouse models. To account for natural progression of microglial activation in the aging brain of rodents [17], we compared standardized differences $(z$-scores) in relation to age among the different mouse models. By this approach, we are able to show for the first time that temporal kinetics of microglial activation differ depending on whether it is driven by tau or amyloid- $\beta$ pathology. In particular, TSPO expression in response to tau pathology showed attenuated and delayed development when compared to TSPO expression in response to A $\beta$ overexpression. Importantly, there were similar increases of the amounts of AT8 positive tau in P301S mice or fibrillar A $\beta$ in APP/PS1 and $A p p^{N L-G-F}$ mice with age, indicating that the observed differences were not driven by variant time courses of protein accumulation. In the translational aspect, the onset of $A \beta$ and tau aggregation may precede the start of clinical symptoms in human $\mathrm{AD}$ [29], and associations of both proteins with microglial activation have already been shown in human PET studies [6]. Thus, the presence of amyloid- $\beta$ and tau should be considered (i.e., by PET or CSF) when interpreting time courses of microglial activation in human neurodegenerative disease, to avoid bias arising from differences in their temporal associations. Furthermore, more detailed studies comparing tau and amyloid- $\beta$ mouse models employing next generation sequencing or proteomics should resolve possible differences of microglia phenotypes in relation to the two abnormal protein aggregates.

Details of the role and time dependence of neuroinflammation in $\mathrm{AD}$ remain a matter of controversy and debate, given the ambivalence of protective and detrimental aspects $[3,28,30]$. This also accounts for some 

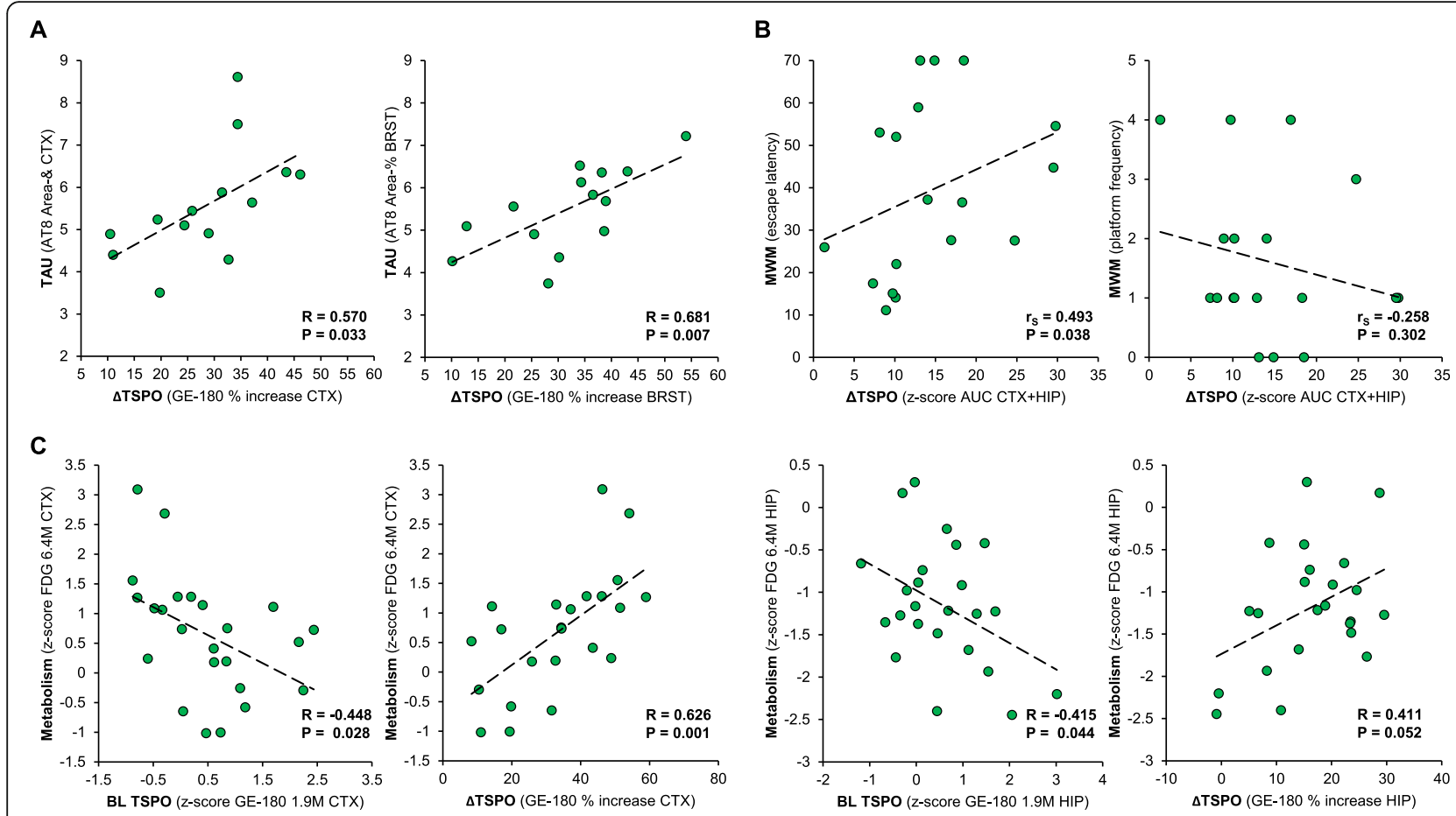

Fig. 5 Associations of early and longitudinal microglial activation with outcome parameters. a Correlation of longitudinal TSPO expression in P301S mice (expressed as \%-increase between 1.9 and 6.4 months of age) with accumulation of AT8 positive tau accumulation in IHC for the cortex and the brainstem; $n=14$. b Correlation of combined longitudinal TSPO expression in cortex and hippocampus (calculated as AUC) with terminal MWM performance; $n=18$. c Correlations of early and longitudinal TSPO expression (BL $z$-score and \%-increase) with terminal glucose metabolism (FDG PET $z$-score) for the cortex and the hippocampus; $n=24$

earlier findings on microglial function in the P301S mouse model. Luo et al. [14] showed in vitro and ex vivo the capability of isolated wild-type microglia to phagocytize tau in brain tissue of P301S mice, implicating a possible protective effect of fully functional microglia. On the other hand, a recent study of Brelstaff et al. [15] indicated that activated microglia can phagocytize neurons of P301S mice and therefore seemingly mediate deleterious effects. This matches with the setting that microglial cells can generally be separated into two classes, proinflammatory (mainly detrimental) and antiinflammatory (mainly protective) phenotypes, and can presumably change from one state to another depending on the required function [28].

The strength of our data lies in its longitudinal in vivo design, covering approximately three quarters of the 9month lifespan of P301S model mice. The compilation of data indicates that higher longitudinal TSPO expression in P301S mice predict higher tau accumulation and worse spatial learning at 6.4 months of age. Interestingly, the corresponding associations with glucose metabolism to ${ }^{18} \mathrm{~F}$-FDG $\mu$ PET gave different predictions for baseline and longitudinal measures. While early elevation in TSPO expression was associated with hypometabolism at 6.4 months, we observed higher terminal glucose metabolism in tau mice with TSPO increasing over time. While the first result suggests an overall deleterious effect of high early TSPO expression on the outcome of P301S mice, the second observation is more consistent with a coupling of microglial activation and glucose metabolism, as observed previously in PS2APP mice [16]. With regard to spatial learning deficits, our earlier study with congruent methodology and design in PS2APP amyloid- $\beta$ mice showed that early microglial activation in the forebrain strongly correlated with better cognitive performance in MWM [31]. Speculatively, this could indicate different predictive capability of TSPO $\mu$ PET depending on whether tau or amyloid- $\beta$ accumulation is the primary driver of microgliosis. Regarding tau mouse models, our observation of higher tau accumulation in mice with early microglial activation is in line with findings of associated tau and neuroinflammation in the forebrain of rTg4510 tau mice [32]. Our data also fit with the observations of attenuated NFT accumulation, reduced neuronal degeneration, and averted cognitive deterioration after pharmacological ablation of senescent microglial and astroglial cells in PS19 mice [33], as well as fitting with the increased tau pathology occurring along with NLRP3 inflammasome activation [7]. In summary, tau-associated microglial activation seems more 
detrimental than amyloid- $\beta$-associated effects. Importantly, our compilation of findings of amyloid- $\beta$ and tau mouse models may help support a model wherein the net effect of neuroinflammation changes from being initially protective $(\mathrm{A}+/ \mathrm{T}-)$ to deleterious in late phases $(\mathrm{A}+/ \mathrm{T}+)$ of $\mathrm{AD}[34]$. Among the limitations of our study, we note that we did not perform longitudinal TSPOPET imaging in male mice. Thus, we cannot evaluate effects of sex on the current results. We acknowledge a gap of 1 week in average between the final PET scan and IHC which might has a limited impact on correlation analyses between both modalities since microglial activation in P301S mice raises with aging.

\section{Conclusions}

Monitoring of microglial activation in P301S tau transgenic is feasible by $\mu \mathrm{PET}$ with the TSPO tracer ${ }^{18} \mathrm{~F}-\mathrm{GE}$ 180 TSPO, as validated by IHC with microglial markers. P301S mice manifest delayed time courses and detrimental associations of their microglial activation with outcome parameters when compared to earlier data of amyloid- $\beta$ mouse models. This should draw further attention to the study of phenotypic differences of the microglial responses to amyloid- $\beta$ and tau accumulation.

\section{Supplementary information}

Supplementary information accompanies this paper at https://doi.org/10. 1186/s12974-020-01883-5.

Additional file 1: Supplemental figure 1. Overview of representative images of IBA1 and CD68 immunohistochemistry $(\mathrm{IHC})$ stainings from 2.76.6 M: The upper row shows representative images of the cortex (CTX), the lower row shows representative images of the brainstem (BRST). Magnification 40x objective/oil; scale Bar: $30 \mu \mathrm{m}$; WT = wild-type; TG = P301S; $M=$ age in months.

\section{Abbreviations \\ Aß: Beta amyloid; AD: Alzheimer's disease; AUC: Area under the curve; BRST: Brainstem; CBL: Cerebellum; CoVs: Coefficients of variation; CSF: Cerebrospinal fluid; CTX: Cortex; $\left({ }^{18} \mathrm{~F}-\right)$ FDG: ${ }^{18}$ F-fluordesoxyglucose; HIP: Hippocampus; IF: Immunofluorescence; IHC: Immunohistochemistry; MAPT: Microtubule-associated protein tau; $\mu$ PET: Small animal positron- emission-tomography; MRI: Magnetic resonance imaging; MWM: Morris water maze; NAC: Nucleus accumbens; NFT: Neurofibrillary tangles; OD: Overall density; p.i.: Post injectionem; $R$ : Pearson's coefficient of correlation; $r_{s}$ : Spearman's coefficient of correlation; SPM: Statistical parametric mapping; SUV: Standardized uptake value; SUVR: SUV-ratio; TSPO: Translocator protein; VOIs: Volumes of interest; WT: Wild-type}

\section{Acknowledgements}

We thank Karin Bormann-Giglmaier and Diana Mahlstedt for excellent technical assistance. The APPPS1 colony was established from a breeding pair kindly provided by M. Jucker (Hertie-Institute for Clinical Brain Research, University of Tübingen and DZNE-Tübingen). App ${ }^{N L-G-F}$ mice were provided by RIKEN BRC through the National Bio-Resource Project of the MEXT, Japan. GE Healthcare made GE-180 cassettes were made available through an earlyaccess model. Florbetaben precursor was kindly provided by Life Molecular Imaging.

\section{Authors' contributions}

FE and CS performed the majority of PET experiments. JML and TB performed immunohistochemistry staining. FE performed Morris water maze testing and analyzing of water maze data. FE, CS, GLB, MD, and MB analyzed and quantified the data. MU, NLA, PB, and AR performed interpretation of the PET data. SL performed and improved radiochemistry. GB rendered expert IT support. AZ, JL, PC, GUH, JH, AR, and MB contributed to the conception and design of the study and interpreted the combined findings. FE and MB wrote the manuscript. BU-S supervised the study as a veterinarian. All authors participated in the generation of the original data, added important intellectual content to the manuscript, and provided critical assessment of the current manuscript. All authors read and approved the final manuscript.

\section{Funding}

The study was financially supported by the SyNergy Cluster (J.H., P.B., C.H., and A.R.) and by the Deutsche Forschungsgemeinschaft (DFG) by a dedicated PET imaging grant to M.B. and A.R. (BR4580/1-1 and RO5194/1-1). G.H. is supported by the German Federal Ministry of Education and Research (01EK1605A HitTau), the NOMIS Foundation (FTLD project).

\section{Availability of data and materials}

The datasets used and/or analyzed during the current study are available from the corresponding author on reasonable request.

\section{Ethics approval}

All experiments were carried out in compliance with the National Guidelines for Animal Protection, Germany, with the approval of the regional Animal Care Committee of the Government of Oberbayern (Regierung Oberbayern) and were overseen by a veterinarian. Animal experiments were conducted in accordance with the guidelines EU Directive 2010/63/EU for animal experiments.

\section{Consent for publication}

Not applicable.

\section{Competing interests}

G.U.H. received research support from GE Healthcare and Neuropore; has ongoing research collaborations with Orion and Prothena; serves as a consultant for AbbVie, AlzProtect, Asceneuron, Biogen, Biohaven, Lundbeck, Novartis, Roche, Sanofi, and UCB; received honoraria for scientific presentations from AbbVie, Biogen, Roche, Teva, UCB, and Zambon; and holds a patent on PERK Activation for the Treatment of Neurodegenerative Diseases (PCT/EP2015/068734). M.B. received speaking honoraria from Life Molecular Imaging and GE healthcare. M.B. is an advisor of Life Molecular Imaging. All other authors report no conflicts of interest.

\section{Author details}

${ }^{1}$ Department of Nuclear Medicine, University Hospital of Munich, LMU Munich, 81377 Munich, Germany. ${ }^{2}$ Center of Neuropathology and Prion Research, University Hospital of Munich, LMU Munich, 81377 Munich, Germany. ${ }^{3}$ German Center for Neurodegenerative Diseases (DZNE) Munich, 81377 Munich, Germany. ${ }^{4}$ German Center for Vertigo and Balance Disorders, DSGZ, University Hospital of Munich, LMU Munich, 81377 Munich, Germany. ${ }^{5}$ Department of Neurology, University Hospital of Munich, LMU Munich, 81377 Munich, Germany. ${ }^{6}$ Munich Cluster for Systems Neurology (SyNergy), 81377 Munich, Germany. Department of Nuclear Medicine, Inselspital Bern, Bern, Switzerland. ${ }^{8}$ School of Psychology and Counselling and $\mathrm{HBB}$, Queensland University of Technology, Brisbane, Australia. ${ }^{9}$ Department of Neurology, Hannover Medical School, Hannover, Germany. ${ }^{10}$ Department of Neurology, Technical University of Munich, Munich, Germany.

Received: 16 March 2020 Accepted: 30 June 2020

Published online: 13 July 2020

\section{References}

1. Serrano-Pozo A, Frosch MP, Masliah E, Hyman BT. Neuropathological alterations in Alzheimer disease. Cold Spring Harb Perspect Med. 2011;1: a006189.

2. Tapia-Rojas C, Cabezas-Opazo F, Deaton CA, Vergara EH, GWW J, Quintanilla RA. It's all about tau. Prog Neurobiol. 2018.

3. Barron M, Gartlon J, Dawson LA, Atkinson PJ, Pardon MC. A state of delirium: deciphering the effect of inflammation on tau pathology in Alzheimer's disease. Exp Gerontol. 2017;94:103-7. 
4. Spillantini MG, Goedert M. Tau pathology and neurodegeneration. Lancet Neurol. 2013;12:609-22.

5. Leyns CEG, Holtzman DM. Glial contributions to neurodegeneration in tauopathies. Mol Neurodegener. 2017;12:50.

6. Dani M, Wood M, Mizoguchi R, Fan Z, Walker Z, Morgan R, Hinz R, Biju M, Kuruvilla T, Brooks DJ, Edison P. Microglial activation correlates in vivo with both tau and amyloid in Alzheimer's disease. Brain. 2018;141:2740-54.

7. Ising C, Venegas C, Zhang S, Scheiblich H, Schmidt SV, Vieira-Saecker A, Schwartz S, Albasset S, McManus RM, Tejera D, et al. NLRP3 inflammasome activation drives tau pathology. Nature. 2019;575:669-73.

8. Edison P, Donat CK, Sastre M. In vivo imaging of glial activation in Alzheimer's disease. Front Neurol. 2018:9:625.

9. Brendel M, Jaworska A, Probst F, Overhoff F, Korzhova V, Lindner S, Carlsen J, Bartenstein P, Harada R, Kudo Y, et al. Small-animal PET imaging of tau pathology with 18F-THK5117 in 2 transgenic mouse models. J Nucl Med. 2016;57:792-8.

10. Allen B, Ingram E, Takao M, Smith MJ, Jakes R, Virdee K, Yoshida H, Holzer $M$, Craxton M, Emson PC, et al. Abundant tau filaments and nonapoptotic neurodegeneration in transgenic mice expressing human P301S tau protein. J Neurosci. 2002;22:9340-51.

11. Woerman AL, Patel S, Kazmi SA, Oehler A, Freyman Y, Espiritu L, Cotter R, Castaneda JA, Olson SH, Prusiner SB. Kinetics of human mutant tau prion formation in the brains of 2 transgenic mouse lines. JAMA Neurol. 2017;74:1464-72.

12. Xu H, Rosler TW, Carlsson T, de Andrade A, Bruch J, Hollerhage M, Oertel WH, Hoglinger GU. Memory deficits correlate with tau and spine pathology in P301S MAPT transgenic mice. Neuropathol Appl Neurobiol. 2014;40:833-43.

13. Bellucci A, Westwood AJ, Ingram E, Casamenti F, Goedert M, Spillantini MG. Induction of inflammatory mediators and microglial activation in mice transgenic for mutant human P301S tau protein. Am J Pathol. 2004;165: 1643-52.

14. Luo W, Liu W, Hu X, Hanna M, Caravaca A, Paul SM. Microglial internalization and degradation of pathological tau is enhanced by an antitau monoclonal antibody. Sci Rep. 2015;5:11161.

15. Brelstaff J, Tolkovsky AM, Ghetti B, Goedert M, Spillantini MG. Living neurons with tau filaments aberrantly expose phosphatidylserine and are phagocytosed by microglia. Cell Rep. 2018;24:1939-48 e1934.

16. Brendel M, Probst F, Jaworska A, Overhoff F, Korzhova V, Albert NL, Beck R, Lindner S, Gildehaus FJ, Baumann K, et al. Glial activation and glucose metabolism in a transgenic amyloid mouse model: a triple-tracer PET study. J Nucl Med. 2016;57:954-60.

17. Brendel M, Focke $C$, Blume $T$, Peters $F$, Deussing M, Probst $F$, Jaworska A, Overhoff F, Albert N, Lindner S, et al. Time courses of cortical glucose metabolism and microglial activity across the life span of wild-type mice: a PET study. J Nucl Med. 2017;58:1984-90.

18. Liu B, Le KX, Park MA, Wang S, Belanger AP, Dubey S, Frost JL, Holton P, Reiser $\mathrm{V}$, Jones $\mathrm{PA}$, et al. In vivo detection of age- and disease-related increases in neuroinflammation by 18F-GE180 TSPO microPET imaging in wild-type and Alzheimer's transgenic mice. J Neurosci. 2015;35:15716-30.

19. Parhizkar S, Arzberger T, Brendel M, Kleinberger G, Deussing M, Focke $C$, Nuscher B, Xiong M, Ghasemigharagoz A, Katzmarski N, et al. Loss of TREM2 function increases amyloid seeding but reduces plaque-associated ApoE. Nat Neurosci. 2019;22:191-204.

20. Sacher C, Blume T, Beyer L, Peters F, Eckenweber F, Sgobio C, Deussing M, Albert NL, Unterrainer M, Lindner S, et al. Longitudinal PET monitoring of amyloidosis and microglial activation in a second generation amyloid-beta mouse model. J Nucl Med. 2019.

21. Deussing M, Blume T, Vomacka L, Mahler C, Focke C, Todica A, Unterrainer M, Albert NL, Lindner S, von Ungern-Sternberg B, et al. Coupling between physiological TSPO expression in brain and myocardium allows stabilization of late-phase cerebral [(18)F]GE180 PET quantification. Neuroimage. 2018; 165:83-91.

22. Focke C, Blume T, Zott B, Shi Y, Deussing M, Peters F, Schmidt C, Kleinberger G, Lindner S, Gildehaus FJ, et al. Early and longitudinal microglial activation but not amyloid accumulation predicts cognitive outcome in PS2APP mice. J Nucl Med. 2019;60:548-54.

23. Sawiak SJ, Wood NI, Williams GB, Morton AJ, Carpenter TA. Voxel-based morphometry in the R6/2 transgenic mouse reveals differences between genotypes not seen with manual 2D morphometry. Neurobiol Dis. 2009;33:20-7.
24. Blume T, Focke $C$, Peters F, Deussing M, Albert NL, Lindner S, Gildehaus F-J, von Ungern-Sternberg B, Ozmen L, Baumann K. Microglial response to increasing amyloid load saturates with aging: a longitudinal dual tracer in vivo $\mu$ PET-study. J Neuroinflammation. 2018;15:307.

25. Tan YL, Yuan Y, Tian L. Microglial regional heterogeneity and its role in the brain. Mol Psychiatry. 2020;25:351-67.

26. Maeda J, Zhang MR, Okauchi T, Ji B, Ono M, Hattori S, Kumata K, Iwata N, Saido TC, Trojanowski JQ, et al. In vivo positron emission tomographic imaging of glial responses to amyloid-beta and tau pathologies in mouse models of Alzheimer's disease and related disorders. J Neurosci. 2011;31: $4720-30$.

27. Yoshiyama Y, Higuchi M, Zhang B, Huang SM, Iwata N, Saido TC, Maeda J, Suhara T, Trojanowski JQ, Lee VM. Synapse loss and microglial activation precede tangles in a P301S tauopathy mouse model. Neuron. 2007;53:337-51.

28. Edison P, Brooks DJ. Role of neuroinflammation in the trajectory of Alzheimer's disease and in vivo quantification using PET. J Alzheimers Dis. 2018;64:S339-51.

29. Jack CR Jr, Bennett DA, Blennow K, Carrillo MC, Dunn B, Haeberlein SB, Holtzman DM, Jagust W, Jessen F, Karlawish J, et al. NIA-AA research framework: toward a biological definition of Alzheimer's disease. Alzheimers Dement. 2018:14:535-62.

30. Chaney A, Williams SR, Boutin $H$. In vivo molecular imaging of neuroinflammation in Alzheimer's disease. J Neurochem. 2019;149:438-51.

31. Focke C, Blume T, Zott B, Shi Y, Deussing M, Peters F, Schmidt C, Kleinberger G, Lindner S, Gildehaus FJ, et al. Early and longitudinal microglial activation but not amyloid accumulation predict cognitive outcome in PS2APP mice. J Nucl Med. 2018.

32. Ishikawa A, Tokunaga M, Maeda J, Minamihisamatsu T, Shimojo M, Takuwa H, Ono M, Ni R, Hirano S, Kuwabara S, et al. In vivo visualization of tau accumulation, microglial activation, and brain atrophy in a mouse model of tauopathy rTg4510. J Alzheimers Dis. 2018;61:1037-52.

33. Bussian TJ, Aziz A, Meyer CF, Swenson BL, van Deursen JM, Baker DJ. Clearance of senescent glial cells prevents tau-dependent pathology and cognitive decline. Nature. 2018;562:578-82.

34. Fan Z, Brooks DJ, Okello A, Edison P. An early and late peak in microglial activation in Alzheimer's disease trajectory. Brain. 2017;140:792-803.

\section{Publisher's Note}

Springer Nature remains neutral with regard to jurisdictional claims in published maps and institutional affiliations.

Ready to submit your research? Choose BMC and benefit from:

- fast, convenient online submission

- thorough peer review by experienced researchers in your field

- rapid publication on acceptance

- support for research data, including large and complex data types

- gold Open Access which fosters wider collaboration and increased citations

- maximum visibility for your research: over $100 \mathrm{M}$ website views per year

At BMC, research is always in progress.

Learn more biomedcentral.com/submissions 